\title{
Le développement d'une économie des singularités dans le champ de l'action sociale et médico-sociale en France : un nouveau champ de questionnements éthiques
}

\author{
Didier Benoit ${ }^{\mathrm{a}}$
}

RÉSUMÉ. Le secteur social et médico-social en France doit faire face à des besoins sociaux qui évoluent rapidement et profondément. La transformation du secteur, orchestrée par les politiques publiques, s'organise autour du développement d'une économie de prestations, censée s'adapter au traitement de besoins de publics fragilisés, notamment par l'âge et les handicaps. Ce marché rassemble des services professionnels personnalisés, susceptibles de répondre aux particularités des personnes qui le sollicitent. Cette transformation s'accompagne de produits et services singuliers, incommensurables, dont le « consommateur » est guidé pour faire le meilleur choix possible. Cette quête est peu évidente, car elle relève d'un marché opaque et incertain quant à la qualité des prestations. Pour pallier ce défaut, le «consommateur» est obligé de recourir à des méthodes d'information lui permettant d'orienter son choix. L'économie des singularités, théorie développée par Karpik (2007), offre une lecture sociologique intéressante du développement du marché prestataire dans le secteur social et médico-social, et dispose d'outils d'analyse rendant intelligible la réalité de cette évolution.

ABSTRACT. In France, the social and medico-social sector is having to meet social needs that are in the process of rapid and profound change. The transformation of this sector, which has been orchestrated by public policies, is coalescing around an economy of benefits that is supposedly in the process of adapting to meet the needs of those groups deemed vulnerable as a result of old age or handicap. This economy or marketplace has been drawing together personalised professional services seen as capable of responding to the individual needs of those requesting them. It consists of distinct products and services that are both unrelated and difficult to evaluate, with the 'consumer' having to be guided through it to make the best possible choice. How to undertake a search for the right product is by no means an obvious matter, as the market is lacking in transparency and its products are of uncertain quality. To reduce the impact of these shortcomings, 'consumers' are forced to have recourse to information-seeking methods in finding a path towards a viable choice. The economics of singularities, a theoretical approach developed by Karpik (2007), offers us an interesting sociological reading of the development and growth of the services market in the social and medico-social sector, and provides us with analytical tools that allow us to grasp the realities of this evolution.

\section{Introduction}

L'économie des singularités, théorie développée par l'économiste-sociologue Lucien Karpik (2007), peut contribuer utilement à la restructuration de l'économie de l'action sociale et médico-sociale. La logique prestataire, qui pénètre aujourd'hui de plus en plus fortement cette action, tant en France qu'au Québec, s’imprègne de ce type d'économie. L'entreprise peut paraitre audacieuse d'interroger cette économie, vouée en priorité à l'humain, selon une analyse destinée à l'économie classique. La question est d'un intérêt réel, l'action sociale possédant une dimension économique qui repose, avant tout, sur des prestations assurées par des professionnels aux compétences diversifiées auprès de publics dépendants. Cette théorie a le mérite d'interroger l'économie du secteur social et médico-social dans ses fondements

${ }^{a}$ Docteur en droit privé - sciences criminelles, Institut du travail social de Lorraine (France) 
sociologiques, ne se limitant pas, ainsi, à une simple approche néo-classique ${ }^{1}$ d'une économie fixant la reconnaissance des produits par le prix comme résultante du jeu de l'offre et de la demande.

En effet, cette modalité de détermination de la valeur d'un produit de consommation est loin de correspondre aux seules attentes des consommateurs. Le prix est loin d'être l'unique facteur déterminant du choix d'un produit. Les produits proposés par le secteur social et médico-social, ces services professionnels, assimilés à des singularités, sont souvent marqués par l'incertitude de leur qualité. Le consommateur dispose de peu d'informations. Se posent les questions de savoir quels sont les logiques, les raisonnements, les moyens qui amènent le consommateur à effectuer le choix de tel bien ou de tel service.

L'action sociale et médico-sociale constitue un marché où se déroule un jeu d'acteurs (opérateurs, autorités tarificatrices, tutelles, consommateurs) animé par des enjeux concurrentiels. Il s'agit notamment de savoir quel acteur, pour tel type de prestation, se montre aujourd'hui le plus performant, considérant la qualité de la prise en charge et son coût. Les orientations marchandes de la gestion de l'action sociale et médico-sociale, souvent imposées par les politiques publiques, viennent renforcer cette interrogation de l'économie des singularités, en particulier dans le champ de la prise en charge des personnes âgées et des services à domicile. Ce marché de services personnalisés et de prestations différenciées relève d'une pluralité de qualités échappant à une réalité objective de classement. Ce marché est fondé sur l'incertitude et l'opacité. Le client ne peut savoir a priori quelle est la meilleure prestation offerte. Après avoir défini l'économie des singularités et les logiques qui la régentent, nous décrypterons la nature économique du secteur social et médico-social. Il importera ensuite d'expliciter les principaux concepts de l'économie de singularités appliqués à l'économie du secteur, avant de s'intéresser à l'exemple particulier de la formulation du choix d'un établissement d'hébergement pour personnes âgées dépendantes (ci-après appelée EHPAD) ${ }^{2}$. Nous conclurons notre propos par une approche de la désingularisation marquée par une perte de la qualité réorientant le marché à une appréciation par les prix.

\section{L'économie des singularités : une approche sociologique de l'économie}

Le premier objectif du consommateur est de vouloir faire le bon choix pour un produit qu'il entend acquérir ou une prestation dont il veut bénéficier. Mais, quelle information sérieuse possède-t-il pour opérer ce choix? La question se pose en particulier pour les biens et services qui revêtent des caractères singuliers. Lorsque le consommateur cherche un bon roman, un bon vin, un bon restaurant, un bon médecin, un bon avocat, il ne doute pas de la connaissance sérieuse qu'il estime posséder pour valider son choix. Pourtant, ces produits culturels, ces prestations professionnelles personnalisées, plus généralement tous ces produits et prestations se définissant de manière singulière, sont caractérisés par une valeur symbolique et par l'incertitude de la qualité. Comment le consommateur peut-il établir cette qualité sinon par un processus de jugement basé sur différents dispositifs qui doivent, sur le principe, lui permettre d'établir le bon choix et lever l'incertitude d'un marché opaque dont relèvent ces biens et services singuliers. L'opacité du marché se repère par la primauté de la concurrence symbolique, par la qualité sur la concurrence matérielle, par le prix, d'où toute l'importance de l'information dont le consommateur doit disposer. De quelles rationalités régulatrices dépend le marché de ces biens et services dont les singularités leur confèrent une valeur aléatoire? L'économie des singularités, comme système d'analyse, propose de rendre compte des transactions portant sur ces biens multidimensionnels et de qualité incertaine, ce que Karpik appelle les produits singuliers (biens et services). Ceux-ci sont «des entités incommensurables : ils sont caractérisés par des constellations de qualités ou de dimensions dont les significations sont inscrites dans leurs relations mutuelles » (Karpik, 2007, p. 39)3.

Ainsi, pour une même qualification, il est possible d'aboutir à autant d'interprétations qu'il y a de consommateurs, chacune débouchant sur une requalification du bien ou service. Ces interprétations multiples, configurant la qualité du produit, en font des produits multidimensionnels (une prestation peut être appréciée de différentes manières selon des critères très différenciés, très personnels). C'est ainsi qu'à l'inverse de biens standards, la concurrence par les prix est moins déterminante que la concurrence par la qualité. Malgré l'opacité du 
marché 4 des biens singuliers liée à leur multidimensionnalité, aux multiples critères de jugement et d'interprétation, au relativisme des moyens devant aider au jugement, le consommateur doit effectuer un choix. Il peut se référer à nombre d'outils qui lui permettent d'objectiver son choix en fonction de ses attentes, de ses objectifs, de ses besoins. Or, les théories néo-classiques du marché considèrent trop souvent qu'une approche homogène des produits corrèle un principe d'incertitude sur la qualité de ces derniers. Il existe donc bien un univers spécifique d'échanges, celui des biens singuliers, que la théorie néo-classique n'appréhende que partiellement par une analyse de la régulation du marché par le prix. Karpik élabore ainsi une boîte à outils permettant de comprendre tout cet ensemble de transactions, les dispositifs de jugement personnels et impersonnels et de confiance devant l'y aider. «Ensemble, ils forment un univers varié, dense, censé procurer aux consommateurs une connaissance suffisante des singularités pour faire des choix raisonnables. Leur efficacité varie avec la crédibilité des connaissances proposées, laquelle dépend elle-même de la confiance portée aux dispositifs de jugement » (Karpik, 2007, p. 43).

Le jugement, contrairement à la décision qui suppose un choix dans un rapport d'équivalences pour un même produit, permet de faire des choix entre des entités incommensurables. Les dispositifs de jugement (appellation, certification, titres professionnels, marque, critiques, guide, classements, présentation) et de confiance (" pour être efficace, le dispositif de jugement doit être crédible. Et pour l'être, il doit bénéficier de la confiance de ceux qui l'utilisent. Le jugement et la confiance sont, ainsi, les deux faces de la même réalité » Karpik, 2007, p. 82) permettant de dissiper l'opacité du marché. Ils proposent aux acheteurs la connaissance qui doit leur faciliter la détermination des choix raisonnables ${ }^{5}$. L'objectif est de faire disparaittre le déficit cognitif ou encore d'inspirer la confiance qui caractérise les consommateurs sur le marché des singularités. C'est aussi pour cette raison que s'ajoute aux obligations contractuelles une déontologie professionnelle afin d'assurer la confiance du public. Les notions de jugement, de dispositif de jugement et de confiance font naitre un être de raison, l'homo singularis ${ }^{6}$, dont les comportements rendent intelligible le fonctionnement de la coordination économique. Pour le choix d'un «bon» restaurant, celui-ci va pouvoir s'appuyer sur ces «machines à papier » que sont les guides Michelin et Parker. Ceux- ci dynamisent leur marché respectif selon des régimes de coordination, appelés régime de l'authenticité ou de l'opinion experte. Ces outils développent ainsi une logique de raréfaction basée sur la prolifération des produits liée à leur différenciation, au développement des marchés de niches et à l'activisme réflexif des consommateurs. Pour l'économie des singularités, les problématiques du marché ne portent pas sur la rareté d'un produit mais sur les problèmes de construction et de prolifération de la rareté?.

Le consommateur disposant d'outils destinés à l'analyse des marchés de produits singuliers, il lui faut alors assurer une coordination de ces outils afin de procéder à ce qu'il considèrera comme son meilleur choix. Le régime de coordination est ainsi composé selon Karpik de trois entités nécessaires à l'ajustement de ce choix : le produit qualifié, le consommateur et les dispositifs de jugement ${ }^{8}$. Cette classification repose sur les distinctions issues de dispositifs impersonnels qui n'impliquent pas de connaitre quelqu'un pour acquérir la connaissance afin de trouver le bon produit, mais peut reposer sur une connaissance à distance - par la lecture d'un guide par exemple - et sur des dispositifs personnels quand, à l'inverse, accéder à cette connaissance nécessite de passer par des relations personnelles. Concernant les dispositifs impersonnels, l'auteur distingue selon l'étendue du marché: un marché restreint comme celui des grands vins de Bourgogne ne repose pas sur les mêmes dispositifs qu'un marché étendu comme celui du prêt-à-porter de luxe. Il distingue ensuite des dispositifs qui analysent le contenu des singularités (le guide Hachette des vins) et ceux qui classent les singularités entre elles (les prix littéraires, les hit-parades). Il distingue enfin les formes d'engagement des consommateurs, selon que celui-ci, actif et autonome, cherche à satisfaire des goûts personnels affirmés ou, inactif, suit les tendances proposées par les dispositifs.

Karpik aboutit à une typologie de sept régimes de coordination: quatre s'appuient sur les dispositifs impersonnels et trois sur les dispositifs personnels. Dans les dispositifs impersonnels figurent le régime de l'authenticité, comme le marché des grands vins, marché restreint et consommateur actif, le régime méga, comme l'industrie du luxe ou celle du cinéma des blockbusters qui doivent intégrer les contraires que sont le volume et la rareté, faire tenir ensemble critères esthétiques et de rentabilité, le régime de l'opinion experte, comme sur un marché restreint quand un con- 
sommateur s'en remet à des dispositifs de prescription comme les prix littéraires, enfin celui de l'opinion commune, à l'exemple du hit-parade dans l'industrie des variétés (Zalio, 2007). Les régimes à dispositifs personnels quant à eux, concernent les objets personnalisés, les produits des quasi-marchés, les services des quasi-professions dont les échanges sont assurés par des réseaux ${ }^{9}$. Ils inscrivent le consommateur dans un marché-réseau, considéré comme le socle commun de tous les marchés de services personnalisés. Les marchés-réseaux sont encastrés dans des dispositifs personnels qui se diversifient en réseau cognitif, réseau marchand et réseau corporatif. Le réseau cognitif repose sur le réseau personnel : famille, amis, "collègues", comme sur les réseaux professionnels, d'usagers ou de consommateurs. Le réseau marchand est constitué de vendeurs et d'acheteurs. Le réseau corporatif comprend des praticiens dont les relations interpersonnelles assurent la circulation d'une connaissance crédible sur les pratiques professionnelles ${ }^{10}$. Ainsi, «le marché-réseau, défini par l'imbrication des réseaux cognitifs, des réseaux marchands et des réseaux corporatifs, comprend, à lui seul, toutes les ressources nécessaires au fonctionnement durable d'un marché de services personnalisés » (Karpik, 2007, p. 138) ${ }^{11}$. Les marchés des services personnalisés reposent sur trois régimes de coordination personnels : le régime des convictions, le régime professionnel et le régime inter firmes. Le régime des convictions opère selon deux logiques: celle des passions communes, à l'exemple du choix de mon violon parce que je partage les mêmes passions que mon luthier, celle des thaumaturges, portant sur des services personnalisés ambigus dont la validité et le sérieux ne sont pas évidents sans que leur valeur soit réellement suspectée. Le régime professionnel repose sur la logique de l'excellence. L'enjeu est de pouvoir choisir le meilleur médecin, le meilleur avocat, en fait, la meilleure prestation. Le régime inter firmes porte sur l'autonomisation d'un marché au sein de la coopération entre les firmes : il englobe la sous-traitance et le partenariat.

L'économie des singularités ne vise pas à substituer aux théories de l'économie néo-libérale une nouvelle théorie économique générale. C'est d'autant moins le propos que l'économie standard ne se désintéresse nullement des singularités, mais n'y accorde qu'une attention secondaire. L'économie des singularités a pour mérite de réintroduire la dimension des sciences humaines, par l'intermédiaire de la sociologie, dans l'analyse de la compréhension de phénomènes de consommation. Pour cela, elle cherche à établir une théorie du choix et du comportement appliquée au consommateur. Elle n'est pas détachée de l'économie standard qui intègre aussi, dans une acception non basique, des dimensions singulières. Son mérite réside dans le recours au jugement qui n'est que l'expression d'une consommation singulière. Les prestations assurées dans le secteur social et médico-social s'accordent à cette expression.

\section{L'action sociale et médico-sociale est une économie d'essence singulière}

L'action sociale et médico-sociale, comme économie de prestations censée répondre aux besoins d'une population aux caractères sociaux déterminés, s'inscrit dans les logiques d'un marché des singularités. Selon l'article L 116-1 du Code français de l'action sociale et des familles (ci-après désigné CASF), l'action sociale et médico-sociale [...] tend à promouvoir, dans un cadre interministériel, l'autonomie et la protection des personnes, la cohésion sociale, l'exercice de la citoyenneté, à prévenir les exclusions et à en corriger les effets. Elle repose sur une évaluation continue des besoins et des attentes des membres de tous les groupes sociaux, en particulier des personnes handicapées et des personnes âgées, des personnes et des familles vulnérables, en situation de précarité ou de pauvreté, et sur la mise à leur disposition de prestations en espèces ou en nature. Elle est mise en œuvre par l'État, les collectivités territoriales et leurs établissements publics, les organismes de sécurité sociale, les associations ainsi que par les institutions sociales et médico-sociales au sens de l'article L. 311-1.

Rien à la lecture de cette définition ne laisse supposer que l'action sociale pourrait obéir à des lois mêmes particulières d'un marché, haut lieu de rencontre de l'offre et de la demande selon l'acception néo-libérale. Pourtant, en France, il est bien fait référence à la notion de prestations délivrées par des établissements ou services. La personne accueillie a «le libre choix entre les prestations adaptées qui lui sont proposées...». La Loi $n^{\circ} 2002-2$ du 2 janvier 2002 rénovant l'action sociale et médico-sociale (ci-après désignée la Loi du 2 janvier 2002) affirme également de grands principes comme la dignité, l'autonomie et la citoyenneté de la personne reconnue dans ses droits. Elle pose le principe de l'individualisation de la prise en charge de l'usager, corrélé par un principe de liberté de choix. Ces principes, posés par la 
loi, favorisent le développement d'un champ à valeur économique réelle, un espace marchand incontestable devant, en principe, s'allier à la prise en compte de la dimension humaine, de l'exigence de qualité, du respect, de la dignité de l'être humain. Autant de considérations qui ne sont pas évoquées dans un marché standard.

Les références de la loi française aux droits de l'usager et à la notion de prestations délivrées par les établissements et services sont en parfaite adéquation avec l'évolution marchande et consumériste de l'action sociale et médico-sociale. Joël Defontaine défend explicitement ce point de vue, jugeant que la Loi du 2 janvier 2002 «place le secteur dans l'économie de service où l'économique... est un élément qu'on ne peut plus ignorer. Et tout l'enjeu consiste à intégrer les actions sociales et médico-sociales dans cette logique de marchandisation, sans perte d'âme ou d'excès de libéralisme»(Defontaine, 2005).

Cette évolution n'est pas contemporaine de la Loi du 2 janvier 2002. La rationalisation des choix budgétaires $(\mathrm{RCB})^{12}$, élaborée à la fin des années 1970 s'inscrivait déjà dans cette logique. L'évolution marchande et libérale de l'action sociale et médico-sociale s'est manifestée aussi par une évolution sémantique. Le mot « patient » qui renvoyait à une notion de passivité et de dépendance de la personne qui s'en remettait à la toute puissance de l'institution qui la prenait en charge, s'est vu remplacé par le mot « usager». Celuici fait référence à la citoyenneté, à la dignité et à la volonté de la loi de faire de la personne un acteur citoyen de sa prise en charge. La loi fait de l'usager un «acheteur actif» de prestations qui doit être informé, en détail, de la qualité des produits (au sens de l'économie des singularités) qui lui sont proposés. L'usager se trouve interpellé comme client, terme que nous retiendrons pour désigner l'usager comme acteur sur un marché (Roux, 2002)13. Toutes ces logiques de promotion de l'individu et de ses droits, de prestations, d'individualisation et de contractualisation ont obligé une grande majorité d'établissements et services à s'inscrire dans des démarches qualité, directement importées du secteur industriel et marchand.

Dans une même logique, Didier Auzoux (1997) expose un argumentaire mettant les travailleurs handicapés à une place de clients. Il reste conscient de la difficulté de cette démonstration puisque ce
« client», en l'occurrence le travailleur en établissements et services d'aide par le travail (ci-après désigné ESAT), ne peut être libre de ses choix. Il ne peut ni comparer les prestations, ni les choisir. Mais, les travailleurs handicapés [...] bénéficient d'un service d'aide à la personne. Allons plus loin : ils sont la raison d'être des ESAT : un service d'aide adapté à chacune des personnes, dont le travail, c'est-à-dire l'énergie qu'elle mobilise, n'est que l'expression d'un moyen au développement individuel, mais pas une fin (Auzoux, 1997, p. 89-96).

Même si le discours dominant reste axé sur le respect de la personne et la qualité de sa prise en charge, cette approche économique de l'action sociale et médico-sociale vient ainsi interpeller les pratiques professionnelles et le fonctionnement des institutions. Émerge la notion de concurrence entre acteurs, alliée au souci de la qualité pour satisfaire un client et ainsi pouvoir le conserver ${ }^{14}$. La qualité des prestations participe à la réputation de l'institution. Il pourra être tenu compte de celle-ci lors de l'attribution des appels à projet, eux-mêmes inscrits dans une logique purement concurrentielle. Ces concepts de concurrence, qualité, client, satisfaction, préconisés dans le monde de l'entreprise s'imposent de fait dans le secteur social et médicosocial, appuyés par les règles de transformation du secteur. C'est l'illustration de ce que Michel Chauvière (2009) appelle la « chalandisation » du secteur social. Il faut désormais «moderniser» l'action sociale et le travail social, c'est-à-dire rationaliser les fonctionnements, penser produit ou prestation et oublier l'approche clinique, produire mieux en dépensant moins, rechercher les gains de productivité, satisfaire les usagers et d'abord accroître leur confiance, rendre régulièrement des comptes à qui de droit et enfin troquer le Code de l'action sociale et des familles contre celui de la consommation.

Toutefois, l'emploi de ces notions et leur compréhension ne peuvent se faire qu'à la condition d'en dégager une approche particulière pour le secteur, différente de l'acception économique commune. La plupart des personnes prises en charge par l'action sociale et médico-sociale le sont par obligation, conséquemment à un état particulier : mise en danger, vulnérabilité, dépendance. L'objet (la prestation) peut ainsi être dissocié du désir (du client) comme de la satisfaction. La personne prise en charge relève de besoins qui ne concordent pas forcément avec ses propres désirs ou demandes. La 
satisfaction de ceux-ci n'est pas forcément corrélative de ce que lui estimera être redevable de la qualité. D'où la question de savoir si la qualité d'une prestation dans le champ social et médico-social doit avoir pour seul critère la satisfaction du client, le but restant le traitement de la dépendance? La question se pose pareillement pour les prestations assurées par diverses catégories de professionnels qui procèdent du même cadre d'intervention: psychiatres, pédopsychiatres, psychologues, ergothérapeutes, assistantes sociales et éducateurs qui développent leurs activités de plus en plus dans des cadres libéraux, individuellement ou sous forme de cabinets pluridisciplinaires.

Plus qu'une simple chalandisation, la marchandisation du secteur repose sur un développement des normes, de la traçabilité, des certifications et de l'évaluation, toujours dans l'objectif d'un meilleur accompagnement des publics. La démarche qualité inclut désormais la mesure de la satisfaction de la clientèle et la mise en place d'actions d'amélioration continue de la qualité de service. Pour favoriser l'efficacité et la qualité des services, le secteur se voit dans l'obligation d'avoir une approche de rationalisation de ses ressources et d'efficience économique du fait d'un financement contraint. Dans le même temps, les modes de financement évoluent. L'état prévisionnel des recettes et des dépenses (EPRD) se substitue aux budgets prévisionnels traditionnels. Il se caractérise par la prévision des produits qui détermine dorénavant celle des charges. Les établissements sociaux et médico-sociaux doivent participer à des appels à projet et rechercher des partenariats. La recomposition économique du secteur est favorisée par les pouvoirs publics qui n’hésitent pas à déléguer à des opérateurs privés le soin d'assurer et de développer des prestations. Ce raisonnement vaut uniquement pour les créneaux soumis aujourd'hui à la concurrence, à savoir, la prise en charge des personnes âgées (hébergements, services à domicile) et celui de l'insertion par l'activité économique pour un public identifié comme socialement vulnérable. Les secteurs des personnes handicapées et de la protection de l'enfance ne sont que partiellement liés à celui-ci ${ }^{15}$. Mais, il n'est pas exclu qu'à l'avenir, ces secteurs soient également influencés par ces logiques marchandes. La recherche du meilleur choix doit prévaloir malgré une prise en charge contrainte. C'est ainsi que le service de l'Aide sociale à l'Enfance doit s'efforcer de rechercher le meilleur établissement pour accueillir tel enfant ou tel adolescent en fonction de son projet individuel. Les parents d'un enfant handicapé vont rechercher l'établissement qui leur paraîtra le plus en mesure d'assurer une prise en charge de qualité de leur enfant. Les descendants d'un parent dépendant rechercheront le meilleur établissement pour une fin de vie. Tous ces cas de figures sont liés au traitement d'un besoin incontournable qui, en aucun cas, n'exclut l'exigence de la qualité et de la satisfaction du client, la qualité devant être immédiate, la satisfaction pouvant être différée. De ces remarques, il est possible de déduire que l'action sociale et médico-sociale s'imprègne d'une économie des singularités, offrant des prestations singulières, s'inscrivant dans une logique de marché. Ces prestations ont avant tout un caractère singulier, car elles relèvent d'une pluralité de qualités recherchées par les clients soucieux de la satisfaction de leurs besoins ou désirs.

\section{L'action sociale et médico-sociale devient un marché de prestations de biens et services singuliers}

Partant des concepts fondateurs de l'économie des singularités, il s'agit de montrer comment ces derniers prennent sens dans le champ de l'économie de l'action sociale et médico-sociale.

Le consommateur. Le mot consommateur a été la première désignation de la personne relevant de l'action sociale. Les professionnels du secteur social et médico-social éprouvant une certaine réticence envers l'économie, le terme consommateur s'est trouvé abandonné au profit du terme bénéficiaire, puis de celui d'usager pour finalement être remplacé par le terme de personne accompagnée. Sous prétexte d'un travail sur l'humain et s'adressant à des personnes vulnérables et dépendantes, la dimension économique de l'action sociale s'est trouvée remise en cause pour ne considérer que l'approche humaine de la personne. La dimension économique de la prise en charge est pourtant bien réelle. Cette approche, revendiquée par les acteurs de terrain, s'est trouvée renforcée par le financement public de l'action sociale et médico-sociale. Cette source de financement est venue déresponsabiliser les acteurs de leurs responsabilités vis-à-vis de la dépense. Aujourd'hui, alors que la source publique se tarit, la considération économique de l'action sociale revient en force. Depuis le début 
des années 2000, les politiques publiques ne cessent de mettre l'accent sur cette dimension, soucieuses de l'efficience du traitement de la question sociale. La reconnaissance des droits des usagers va également dans le sens de l'affirmation du consommateur, distingué dans son individualité.

Certes, la notion de consommateur reste particulière. La personne en situation de dépendance bénéficie d'un exercice trop souvent relatif de sa citoyenneté inscrit dans une relation asymétrique vis-à-vis de l'institution. Il n'en est pas moins vrai que la personne reste consommatrice de biens et services qu'elle est de plus en plus en situation de devoir assumer sur le plan économique. Le statut de consommateur ne se définit pas à partir de son rôle actif ou passif, mais découle tout simplement des logiques d'évolution et de transformation du secteur social vers une véritable économie de prestations, soucieuse de la réduction de la dépense publique par un transfert de charges vers le secteur privé lucratif ou non, ainsi que vers la personne accompagnée. C'est bien la restructuration du secteur social et médico-social, voulue par les politiques publiques, qui transforme, petit à petit, l'usager de ce secteur en consommateur inscrit dans un marché prestataire, reposant avant tout sur l'exigence de la qualité.

Le marché. Le traitement de la question sociale en France a toujours relevé de l'autorité publique, et a été délégué à des acteurs accrédités, qui se sont suffis de la manne publique pour aujourd'hui assurer leurs missions. Poussés par un contexte de déficit public, ces mêmes autorités rationalisent leurs choix budgétaires dans un impératif d'économie. C'est l'occasion pour des acteurs à but lucratif d'investir, sous la bienveillance des autorités publiques, le traitement de la question sociale s'inscrivant dans des champs ou dans des types d'interventions qu'ils pensent être rentables. Nous avons ainsi deux grandes catégories d'acteurs. La première "non lucrative » (acteur historique) doit s'inscrire dans l'efficience économique. La contrainte gestionnaire oblige à l'économie tout en exigeant le maintien de la qualité de l'accompagnement de l'usager. La seconde «lucrative » s'inscrit clairement dans la logique de profit.

C'est un marché qui s'adresse à des publics particuliers, marqués par des caractères de dépendance et de vulnérabilité qui obligent ces derniers à être tributaires d'une offre donnée. Ils dépendent de l'offre existante, de la qualité révélée ou non, du coût à assumer. C’est un marché :

- qui reste tendu de par un déficit chronique de l'offre face à une demande croissante. L'effectif croissant des personnes âgées en EHPAD s'explique par la génération des baby-boomers d'après-guerre et l'allongement de l'espérance de vie. Même si l'âge moyen (86 ans) du placement se trouve plus tardif, la demande reste forte à la suite de la perte rapide de l'indépendance à partir d'un certain âge. L'offre de prise en charge reste tendue du fait de choix politiques orientés vers le maintien à domicile. D'où le choix affirmé, dans les politiques publiques du développement, d'un marché prestataire censé répondre à cette exigence et facilitant le maillage du territoire.

- qui reste très dépendant de la notion de territoire. La notion de besoin et de réponse relativement aux moyens est traitée dans le cadre de schémas départementaux. La notion de territoire est renforcée par la logique du rapprochement familial. Ce zonage géographique fait naitre un public captif, lié lui-même à une situation géographique donnée et à une situation personnelle de nature cognitive et affective.

- qui reste opaque. Les acteurs se dévoilent peu, sinon sur des sites Internet qui ne manquent pas de vanter la qualité de leurs prestations. Mais, est-ce là une information vraiment objective? Son unilatéralité affaiblit l'objectivité de cette dernière. D'où le maintien d'une incertitude sur la qualité des biens et des services dispensés.

Le développement de la prestation présente un caractère ténu de la concurrence dans le secteur social et médico-social. Ce type de marché constitue une incitation pour un nombre de plus en plus élevé d'acteurs à la recherche de profits.

Un marché de biens singuliers. Un bien singulier dont la qualité est la référence est un bien caractérisé par son incommensurabilité et sa multidimentionnalité. Dans le secteur social et médico-social, ces biens, dans l'énoncé du choix, s'inscrivent dans un environnement bien spécifique de la personne, celui de 
son histoire, sa culture, son éducation. C'est avant tout le caractère humain qui se révèle important. Il s'agit d'être respectueux de la personne, sans jugement de valeur.

Ce sont par ailleurs des biens sur lesquels la personne dispose de peu d'informations pour juger de la qualité. D'où un climat d'incertitude quant à l'établissement d'un choix objectif et raisonné. Le choix peut revêtir un caractère opportuniste par manque d'éléments objectifs. Ce déficit oriente vers un choix de confiance où la personne s'en remet à l'opérateur, quitte à déjuger son choix initial par son vécu. C'est alors que se construit le champ des réclamations, des remontrances, des revendications, des doléances, des recours, provoquant le phénomène de judiciarisation du secteur.

L'incertitude de la qualité, par défaut de dispositif de jugement, fait naître le caractère multidimensionnel du bien singulier. Celui-ci s'appuie sur la multiplicité des avis, des interprétations, des opinions, plus ou moins argumentés, rarement vérifiés, voire vérifiables. D'où le bouche à oreille plus ou moins objectif. C'est là tout le caractère relatif, fondé ou pas, de ce dernier. L'incertitude reste difficile à dissiper. Seules l'expérience et la pratique du bien peuvent permettre d'y remédier.

Le jugement comme choix. Comment établir un choix quand la connaissance du lieu, des personnels et de leurs fonctionnements, des services et prestations assurés n'est pas rendue objectivable par des processus de mesure, d'identification de contenu, d'évaluation de leur rendement, de leur pertinence pour ce qui est des besoins identifiés? Le consommateur doit rendre objectivable la démarche de construction de son jugement comme choix, partant de ce qu'il estime relever de son objectivité. La visite du lieu, des échanges avec les personnels, quelques documents de présentation peuvent l'y aider. Il construit son jugement en élaborant une évaluation synthétique qui intègre une pluralité de critères qui l'aide à formuler une opinion propre. C'est de cette opinion, dont l'objectivité peut être remise en cause à tout moment au gré de faits rapportés par l'expérience ou de la survenance d'une information contradictoire, que se cristallise le jugement. Cette pluralité de critères peut, à tout moment, être affectée de pondérations variables qui peuvent confirmer ou infirmer la pertinence du choix, comme l'inférer ou la modérer.
L'existence d'informations objectives n'est pas à négliger. Pour aider à la formalisation du choix d'un EHPAD, il existe des plateformes accessibles par Internet qui donnent une information instantanée sur les places disponibles, dans quels établissements sur tel lieu géographique. Il est aussi proposé, sur le site Internet des EHPAD, un logiciel de calcul qui permet d'évaluer le montant dont devra s'acquitter le consommateur en fonction des aides dont il peut bénéficier. Il est parfaitement clair que l'information la plus objective porte sur les critères de territoire, de disponibilité, de coût. Rien ne permet de disposer d'une information objective sur la qualité des prestations assurées, sinon une information purement déclarative.

Les dispositifs de jugement. Ceux-ci restent embryonnaires dans le champ de l'action sociale et médicosociale. C'est certainement le jeu des relations interpersonnelles qui a le plus d'impact. De nombreux acteurs institutionnels recourent aux labels et normes diverses. Cette démarche garde un caractère récent. Ce n'est qu'après l'usage et le vécu des processus de vérification que ces labels acquerront autorité. Il n'y a pas de cicérones, ni de classements dans le secteur. Pour l'heure, on doit accorder sa confiance à des hebdomadaires comme Le Point ou le Nouvel Observateur qui le pratiquent dans le champ du sanitaire.

Depuis la Loi du 2 janvier 2002, les établissements et services du secteur social et médico-social doivent se conformer à une évaluation interne tous les cinq ans et une évaluation externe tous les sept ans. Mais, qui a connaissance de ces résultats évaluatifs, excepté les autorités de tutelle? Ne serait-il pas pertinent que ces démarches quittent l'intimité des autorités de tutelle et des établissements pour être portées à la connaissance des futurs consommateurs afin de les aider à formuler leur choix? Le plus souvent, d'une connaissance que le consommateur s'est forgée lui-même, naît la confiance empirique portée sur le dispositif de prise en charge. Il est aidé en cela par quelques supports cognitifs, quand ils existent, comme les labels, les connaissances orientées (opinions) bien qu'il soit parfois difficile de donner sens à ces références.

Le régime de coordination impersonnel. On ne peut identifier un quelconque régime de coordination impersonnel dans le quasi-marché que constitue le secteur social et médico-social. Dans ce type de 
régime, la qualification des produits singuliers résulte de la combinaison de la taille du marché, des formes d'intervention, des dispositifs impersonnels et des formes d'engagement des consommateurs. Peut-on, en l'espèce, parler de taille du marché? Le marché est présent sur l'ensemble de l'hexagone pour une offre inégalement répartie sur le territoire. Cette inégalité s'est constituée selon une logique d'implantation des acteurs sollicités par des collectivités territoriales soucieuses de satisfaire à des impératifs économiques. La tendance s'inverse aujourd'hui à la suite d'une forte pression de demandes de consommation devant être satisfaites de manière équitable sur l'ensemble du territoire.

Une évolution contemporaine notable, à savoir l'évolution de la taille des acteurs, est la conséquence de la restructuration du secteur social et médico-social selon une logique de regroupement, fusion, absorption. Figurent sur le marché de petites associations mono établissement et de grosses associations et fondations, qui sont amenées à gérer un nombre conséquent d'établissements et de services. Cette nouvelle structuration des acteurs influence la tension entre le maintien des singularités et la recherche de la rentabilité. Ce n'est pas étonnant qu'au-delà d'une logique d'efficience, des fonds de pensions et de grands groupes constitués investissent ce champ.

Le régime de l'opinion experte n'est pas présent dans le secteur. Ce régime intègre les dispositifs formels et le marché restreint, la passivité et l'autonomie du consommateur autour d'une logique globale de délégation de jugement conditionnelle. Il n'y a pas d'opérations de classements des produits et services singuliers qui reposeraient sur une évaluation spécifique des produits. C'est dans cette dimension que pourraient être exploitées, au bénéfice du consommateur, les évaluations internes et externes.

Le régime de coordination personnel. Ce régime repose sur une coordination principalement établie par un réseau de relations interpersonnelles. Le réseau s'établit de manière informelle comme il peut s'établir de manière structurée (associations de consommateurs). La simple répétition d'une information suffit à instaurer ou non la confiance. La fiabilité de la connaissance repose sur une information qui se trouve confortée dans le temps et non interférée par quelques faits incidents. Ainsi, le régime de coordination personnel le plus commun dans le secteur est celui du régime de l'opinion commune. Il intègre des dispositifs formels (sites Internet), une qualification des prestations par des positions relatives (ce n'est que l'opinion de ...), un consommateur passif et hétéronome, c'est-à-dire un consommateur peu critique sur l'information qui lui est donnée. Le consommateur se satisfait ainsi d'une délégation de jugement fondée sur la commodité.

Le prix. Pour l'économie des singularités, la concurrence par la qualité est une primauté. Pour autant, la question du prix des biens singuliers n'est pas à négliger. La fixation du prix obéit à des règles particulières dans le secteur social et médico-social. L'autorisation de création et de fonctionnement, l'habilitation et la tarification des établissements et services sont organisées par voies législatives et réglementaires et traduisent ces obligations, dans un environnement contraint par des instruments collectifs de régulation : la planification (schémas départementaux, régionaux et nationaux et programmes interdépartementaux d'accompagnement du handicap et de la perte d'autonomie et les enveloppes limitatives de crédits, objectif d'évolution et montant annuel des dépenses prises en compte dans les tarifs, fixés par les ministres, à partir des crédits votés par le Parlement en lois de finances et de financement de la sécurité sociale). Selon la Direction générale de la cohésion sociale (DGCS $)^{16}$, la tarification des établissements et services sociaux et médico-sociaux a été organisée, d'une part, pour garantir les moyens financiers nécessaires aux établissements pour l'accomplissement de leurs missions et, d'autre part, pour éviter aux financeurs et aux usagers (consommateurs) des dépenses injustifiées, voire abusives. Le projet de l'établissement ou service est le fondement de l'argumentaire pour négocier les propositions budgétaires avec les autorités compétentes.

La tarification des établissements et services du secteur social et médico-social s'établit selon deux modalités : la dotation globale de financement ${ }^{17}$ et la fixation d'un prix de journée ${ }^{18}$. Ces modalités d'élaboration budgétaire sont aujourd'hui modifiées par l'introduction d'une nouvelle logique appelée « état prévisionnel des recettes et des dépenses » (EPRD). Il s'agit avant tout d'une inversion de logique par rapport aux conditions d'établissements des budgets prévisionnels passés. On passe d'une logique de transmission à l'Agence 
régionale de Santé (ARS) d'un montant de dépenses qui induit le niveau de ressources (cadres classiques) à une logique d'évaluation de l'activité qui induit un niveau de ressources, qui autorise un degré de dépenses et donc de moyens. Le niveau de responsabilité des organismes gestionnaires en sort renforcé. Il dispose ainsi, sous couvert du code de l'action sociale et des familles (CASF) et des dispositions du contrat prévisionnel d'objectifs et de moyens (CPOM), d'une plus grande liberté en matière de pilotage financier et stratégique des établissements et services sociaux et médicosociaux (ESMS). L'état prévisionnel des recettes et des dépenses (EPRD) a vocation d'être un outil de pilotage interne pour l'ESMS. Les prévisions doivent être sincères et refléter ce qui va se produire dans l'année $\mathrm{N}$ et pour les cinq années suivantes. L'intervention de l'Agence régionale de Santé (ARS) dans la campagne budgétaire est recentrée sur la validation des grandes masses financières, de la bonne trajectoire financière de l'établissement et de la conformité avec les objectifs du CPOM. Ce système est pour l'instant transitoire.

Le consommateur n'est aucunement un acteur dans la fixation du prix qui n'est pas un élément de cristallisation de la concurrence. Ces procédures de fixation sont aujourd'hui essentiellement orientées vers une réduction de la dépense publique, indépendamment de toute prise en considération de la qualité des prestations qui, dans son principe, est maintenue. Certes, en dehors de l'engagement des autorités tarificatrices, un établissement reste libre de développer des prestations, le coût de ces dernières venant en supplément. Ainsi, le coût mensuel d'une prise en charge en EHPAD est d'environ 2000 euros, coût pouvant s'élever jusqu'à 3000, 4000, même 5000 euros mensuellement, en fonction de la qualité estimée des prestations. Ces derniers ciblent des clientèles particulièrement fortunées. Le consommateur, placé dans une situation de dépense du coût de la prestation ne peut faire ses choix qu'en fonction de ses dispositions financières. C'est un élément explicatif de l'âge avancé du placement en EHPAD (en moyenne, 86 ans). Le calcul du placement et ses modalités de financement se font le plus souvent sur une estimation du patrimoine du consommateur. Les livrets de placements financiers sont sollicités. La vente d'un bien immobilier peut-être décidée. La dépense en EHPAD ne relève pas d'un jeu concurrentiel.
Elle réside dans la prévision d’une dépense évaluée sur l'actif d'un patrimoine mobilier et immobilier.

Les opérateurs du marché. le terme opérateur désigne les acteurs institutionnels gestionnaires d'établissements et services quels que soient leurs statuts juridiques : association, fondation, acteurs publics. Le terme acteur désigne l'ensemble des personnes physiques ou morales de droit privé ou public agissant sur le marché. Il s'agit des acteurs associatifs gestionnaires d'établissements et services, des collectivités publiques dans l'exercice de leurs missions, des consommateurs. Les opérateurs sont de statut public pour $30 \%$ ou de statut privé pour $70 \%$. Le secteur de droit privé gestionnaire dans le secteur est presque exclusivement sous statut associatif non lucratif. L'arrivée depuis une quinzaine d'années d'opérateurs à but lucratif est venue fortement perturber les représentations des opérateurs traditionnels. Le clivage supposé entre les deux natures d'opérateurs s'est cristallisé autour de la notion de valeur. Un discours simplifié s'est construit laissant supposer que seuls les opérateurs non lucratifs relevaient des bonnes valeurs françaises, à savoir l'humanisme, la solidarité, la fraternité... Cependant, le secteur lucratif ne manque pas, lui aussi, de revendiquer certaines valeurs, celles-ci participant à la valorisation commerciale de l'institution. Mais, l'avenir du secteur social et médicosocial ne se joue pas à ce niveau. Les opérateurs historiques ne sont pas exempts de tout reproche. Les phénomènes de maltraitance restent encore une réalité dans les pratiques professionnelles malgré tous les efforts déployés par la loi, relayés par les procédures d'évaluation et l'analyse des pratiques professionnelles. Les valeurs sont une chose, mais ce qui prévaut à la restructuration du secteur social et médico-social, c'est son efficience économique. Ainsi, pour réaliser cette efficience et répondre aux exigences d'un marché, les acteurs associatifs gestionnaires développent les mêmes techniques entrepreneuriales que les acteurs lucratifs. «C'est ainsi que les opérateurs du secteur social et médico-social abandonnent progressivement les caractéristiques historiques des établissements traditionnels pour adopter les préoccupations et les techniques de n'importe quelle organisation entrepreneuriale, pour peu qu'on les adapte au contexte et à la vocation des structures qui sont concernées. Car, même si une entreprise de service à caractère social et médico-social ne possède pas de finalité lucrative, elle vise bien une gestion optimale des 
ressources et la meilleure performance possible, même si elle ne souhaite pas absolument conquérir des marchés ou des parts de marché. Elle tend bien à répondre au mieux aux besoins de ses différents clients. Et quand bien même, elle ne se considère pas dans un secteur concurrentiel, elle doit bien soutenir la comparaison avec d'autres entreprises ou dispositifs et se préoccuper de qualité » (Batifoulier, 2011).

Il n'est pas inutile non plus de rappeler qu'en fonction du droit communautaire, "une association sans but lucratif exerçant une activité économique d'intérêt général et sollicitant un concours financier sera qualifiée d'entreprise au sens européen et soumise à la réglementation des aides d'État pour son activité économique » (Laurent, 2010) ${ }^{19}$. La notion d'activité économique recouvre, quel que soit le secteur d'activité, toute offre de biens ou de services sur un marché donné. La nature sociale de l'activité n'est pas, en soi, suffisante pour faire exception à la qualification d'activité économique. De même, les activités d'une association, susceptibles de bénéficier du concours public et ne poursuivant pas un but lucratif, ne sont pas pour autant de nature non économiques. Répondant aux besoins de consommateurs dans un cadre contractualisé, cadre imposé par la Loi du 2 janvier 2002, les opérateurs associatifs non lucratifs s'apparentent, de plus en plus, à des entreprises inscrivant leurs activités dans des logiques de marché.

\section{Le marché de l'action sociale et médio-sociale : un marché basé sur le modèle de la personnalisation et de l'information imparfaite}

De l'analyse du marché et des principaux concepts qui le régissent, il apparait clairement que ce dernier est fortement marqué par la personnalisation des biens et services et par un déficit récurrent d'information. C'est donc un marché qui se trouve fortement dépendant de la personne même du consommateur, en tant qu'être humain singulier, en demande de prestations adaptées à sa situation de dépendance, et des acteurs professionnels relevant de qualités humaines et professionnelles différentes. Certains se révèlent humains, d'autres moins. Certains font preuve d'une grande professionnalité, d'autres pas. Par ailleurs, le consommateur n'est pas un être unique, prototypé. Il est avant tout un être singulier de par son degré de dépendance et de vulnérabilité, ce qui ne l'empêche pas d'adopter des comportements très différents selon les intervenants (respect, non-respect, agressivité, violence...), pouvant contester la qualité de la prestation même si celle-ci est présente. C'est la dimension de l'être, sa personnalité qui, avant tout, est mise en avant dans l'acte de consommer la prestation. Certains cherchent à lier une relation sympathique avec le professionnel. D'autres affichent la mentalité du consommateur stricto sensu : « je paie, donc j'ai droit». La situation est également complexe pour le professionnel. Inscrivant sa prestation dans un rapport humain, il est forcément plus sensible à tel ou tel consommateur pour lequel il fait preuve d'une plus grande empathie. Il est évident que pour une même prestation, la qualité se révèle inégale selon le professionnel. C'est très souvent la dimension du contact humain avec l'usager qui emporte la différence.

C'est également un marché marqué par des biens et services relevant d'une information imparfaite. La qualité est difficilement vérifiable en amont. Le client a une information imparfaite non seulement ex ante (au moment de «l'achat », et donc avant l'usage), mais peut-être aussi expost (après « usage »). La qualité est souvent peu vérifiée en aval. De plus, la dimension humaine du travail social introduit une grande part de subjectivité dans l'appréciation portée sur telle ou telle prestation. Cette dimension se retrouve aussi dans la relation avec le professionnel. Certains clients manifesteront une accroche plus positive avec tel professionnel plutôt qu'avec tel autre. Les biens et services pour lesquels il y a information imparfaite sur la qualité ex ante sont appelés, dans la théorie économique, des «biens d'expérience». Leur qualité se révèle à l'usage, après l'achat. Les biens pour lesquels l'incertitude demeure après l'usage sont nommés « biens de confiance ». La mesure de la qualité repose bien sur l'adéquation de la professionnalité technique des services couplée à des facteurs psychologiques comme la sympathie, la compatibilité d'humeur. C'est pourquoi, les biens et services du secteur social et médico-social ne relèvent pas seulement d'un simple problème d'achat, mais révèle une problématique «d'appariement» (Gautié, 2008). La consommation se faisant majoritairement dans un cadre institutionnel contraignant, l'institution participe aussi à la qualité. La qualité résulte d'une coconstruction entre le consommateur, le professionnel et l'institution. 


\section{Les singularités du choix d'un Établissement d'Hébergement pour Personnes Âgées Dépendantes (EHPAD)}

Choisir un EHPAD n'est pas chose facile. Le choix est conditionné par le souci de la personne âgée d'être placée dans un établissement d'accueil qui soit en mesure d'assurer une qualité de prise en charge pour un coût jugé raisonnable. Le marché des EHPAD reste aujourd'hui investi par des opérateurs divers. Il peut s'agir de petites associations gérant un établissement, comme de plus grosses institutions gestionnaires de plusieurs établissements. Du fait de l'ouverture de ce marché à des opérateurs privés lucratifs, de grands groupes prestataires s'y investissent en créant des chaînes labélisées. Des fonds de pension sont également présents dans ce champ jugé prometteur en matière d'investissements lucratifs. Sur ce marché en pleine expansion, liée notamment à l'augmentation de l'espérance de vie, selon quelles modalités et quels critères, le futur consommateur va-t-il pouvoir faire le choix d'un établissement? (Le consommateur est ici aussi client, considérant que la personne prise en charge est bien celle qui achète et consomme des biens et services en fonction de l'exigence de ses besoins et désirs). Donc, la source d'information s'avère indispensable. Celle-ci peut relever de la connaissance des établissements que la personne peut avoir. Cette source est souvent insuffisante, car très incomplète, voire incertaine. Les situations de placement n'étant généralement pas anticipées, c'est au dernier moment que se fait la recherche de l'information. Le choix est plus conditionné par le traitement d'une situation d'urgence que par la recherche réelle de la qualité. Cette situation est normale. Elle correspond à la réticence des personnes âgées à être placées. Le bouche à oreille peut également fonctionner, mais de manière limitée. Encore faut-il connaitre des personnes qui ont dû gérer des problématiques de placement assez semblables à celles qu'il faut résoudre dans un même contexte géographique. Enfin, Internet est une source d'approche et d'accroche déterminante. Toute association a un site Internet qui vante, bien évidemment, les qualités de la prise en charge comme le bien être, offerts par l'établissement.

Il s'agit aussi pour le consommateur de se poser les bonnes questions afin de pouvoir procéder à un choix raisonnable. Les critères de choix vont dépendre des situations personnelles. Il est néanmoins possible de déterminer des catégories de critères auxquelles doit se référer tout client : la localisation de l'établissement (privilégier le rapprochement familial ou le maintien dans l'environnement de vie actuel), le degré d'autonomie (il existe plusieurs types d'établissements selon le degré de dépendance), le statut de l'établissement privé lucratif (gérés par des sociétés commerciales, privé non lucratif (rattachés à une fondation ou association), ou public (autonomes ou rattachés à un établissement sanitaire ou à une collectivité locale), ainsi que le degré de ressources (établissement habilité ou non à l'aide sociale). Le choix ne peut être définitif sans une visite de l'établissement. C'est l'occasion de juger l'accessibilité de l'établissement, son environnement, la qualité des chambres et des parties communes, et d'échanger avec le personnel. Cette phase doit être pensée comme la réalisation d'un véritable travail où l'activité d'évaluation serait déléguée aux consommateurs. Cette évaluation est dès lors organisée dans un rapport social nouveau créant de la valeur ajoutée pour l'institution (Dujarier, 2014).

Ce panel de critères engendre nombre de questions auxquelles le consommateur doit s'efforcer de trouver des réponses pour opérer le bon choix. Celui-ci va disposer de critères objectifs (tarifs, situation de l'établissement, types de prestations...) qui vont guider son choix sans permettre d'apprécier la qualité réelle de la prise en charge. La qualité des prestations va reposer sur des critères guère mesurables au moment du choix, mais vérifiables dans la prise en charge quotidienne. Ces critères sont, entre autres, l'approche humaine de la personne et le respect de ses droits, le respect des obligations de l'établissement, la qualité des pratiques professionnelles. Partant d'un produit relevant d'une certaine standardisation (les prestations types, le fonctionnement général de l'établissement), il s'agit de déterminer le produit personnalisé ${ }^{20}$ défini par «la référence la plus radicalement opposée à l'interchangeabilité : la personne humaine. Par voie de conséquence, les producteurs, les consommateurs, les biens originaux et les services personnalisés se qualifient mutuellement comme des entités incommensurables » (Karpik, 2007, p. 51) et multidimensionnelles relevant d'une certaine opacité et de la difficulté de juger de la pluralité des qualités. Les qualités vont reposer sur les modes d'intervention des personnels ${ }^{21}$, organisés dans une expertise et une éthique collective, privilégiant l'intérêt du client et la qualité du service rendu avant la recherche du profit quelque soit sa nature. Les prestations personnalisées se diversifient donc « en fonction de l'intensité des relations et des 
interactions entre les acteurs de l'offre et de la demande » (Gadrey et Bandt, 1994), une demande obligée (le client se doit de recourir à la prestation) pour une offre contrainte (dimension institutionnelle de l'offre, contrainte budgétaire). Les prestations assurées dans le cadre de la prise en charge d'une personne âgée sont donc des produits singuliers, relevant d'une équation dont les deux membres sont les suivants : l'intervention la plus ajustée possible aux besoins, demandes, désirs spécifiques des personnes prise en charge (qualité, satisfaction) et les contraintes des établissements (légales et réglementaires).

Certains critères sont objectivement mesurables en amont et déterminent le choix. Celui-ci procède du calcul que représente l'appréciation cumulée des critères objectifs. Il ne porte que sur une appréciation partielle du «bon» EHPAD. L'addition de ces critères objectifs constitue une information crédible avec la durée de la pratique et permet alors de formuler un jugement, c'est-à-dire, un choix qualitatif, fondé sur la relation entre des critères d'objectivation et l'évaluation des besoins de la personne ${ }^{22}$. Ce jugement viendra confirmer ou infirmer le choix initial, avec pour conséquence éventuelle le dépôt de réclamations, de plaintes, voire le retrait de la personne placée. L'assurance de la qualité n'est pas garantie seulement par les critères objectifs, ceux-ci n'intégrant pas, ou trop peu, la dimension humaine de la prise en charge, source éventuelle d'insatisfaction, voire de déception, pouvant contredire le choix initial. Nous nous situons bien là dans une dimension incommensurable et multidimensionnelle du produit personnalisé. Se pose alors la nécessité de définir les critères d'évaluation possibles de la dimension humaine de la prise en charge, d'évaluer le rapport humain entre les catégories de personnels et le client, qui peuvent s'apprécier différemment selon les pratiques professionnelles concernées. Il s'agit du respect des droits du consommateur, reposant sur un droit non plus déclaratif comme spécifié dans la Loi du 2 janvier 2002, mais dont le sens doit être décliné en fonction de la personne. Le respect d'une éthique participe également à cette qualité. Il ne faut pas oublier que ce critère de la qualité de la relation reste tributaire du rapport entre le professionnel et le consommateur, dans ce qu'il recouvre de morale et de fierté dans les pratiques $^{23}$. La qualité peut être remise en cause, à tout moment, avec la modification du comportement d'un professionnel ou le départ de celui-ci. Plus communément, le souci de la qualité de la prise en charge du consommateur se réfère à des préconisations formulées par la haute autorité de santé (HAS) ${ }^{24}$, de bonnes pratiques professionnelles, comme moyen de prévenir les situations de maltraitance. L'implication professionnelle de chacun doit donc respecter des références techniques, méthodologiques et éthiques devant faire l'objet d'une évaluation régulière. Ces concepts appartiennent à la culture du monde de l'entreprise, plus généralement, du secteur lucratif. Pour le secteur social et médico-social, les bonnes pratiques professionnelles visent principalement l'éthique, la qualité des prestations, plus généralement, le bien-être de la personne. La HAS définit une «bonne pratique professionnelle» comme étant une action qui s'appuie sur des « recommandations (sens lié à une «plus value» pour l'usager), traduites dans des références (exigence affirmative et positive en ce qui a trait aux objectifs à atteindre) déclinées dans des procédures (séquence ordonnée d'activités ou d'interventions mises en œuvre dans une situation définie) 》 (Gacoin, 2007). Les consommateurs peuvent toujours consulter le site de l'HAS pour prendre connaissance de ces recommandations. Mais, l'existence de celles-ci interpellent avant tout les professionnels comme un outil de réflexion pour développer des dynamiques de qualité portées par des équipes tant dans l'énoncé de procédures ou de «normes», que dans l'inscription des équipes dans des démarches évaluatives. Dès lors, le caractère incommensurable du produit personnalisé repose sur la qualité de celuici au sens de la sécurité, de la qualité du service rendu et de la qualité perçue par le client et son entourage, de la qualité des soins, de l'équité et de l'éthique, de la qualité humaine et technique des pratiques professionnelles, de la capacité de réactivité et d'adaptation de ces derniers. Son caractère multidimensionnel se réfère au prix, aux attributs techniques et matériels, à la catégorie, à la qualité perçue ou comprise, à la notoriété acquise. Cette analyse du produit personnalisé met à jour une mesure de la performance de ce dernier et le développement d'approches comparatives.

Pour arrêter le choix d'un «bon» EHPAD, l'usager ou le client doit surmonter l'incommensurabilité et la multidimensionnalité des termes du choix. Pour cela, il dispose de critères objectifs qui participent à la connaissance du produit lui permettant de qualifier celuici. L'addition de ces critères lui permet de formuler un jugement. L'addition de critères subjectifs peut l'amener à la reformulation du jugement premier qui peut conduire à une requalification du produit en fonction du degré de satisfaction ou d'insatisfaction éprouvé. 
Le jugement reste principalement établi sur des critères qui permettent de formuler un jugement révisable, de qualifier le produit en adéquation avec les besoins du consommateur. Ces critères s'établissent à partir de dispositifs de jugement ayant pour support les sites Internet et l'existence de réseaux personnels ou professionnels. Néanmoins, les sites Internet proposent une connaissance orientée du produit, participant à la construction d'un idéal de prise en charge ${ }^{25}$, mais, néanmoins faisant état des possibilités réelles de celle-ci. Ils construisent la relation d'échange entre les acteurs de la prise en charge et le consommateur, notamment dans l'espace de négociation qu'est la rédaction du contrat de séjour dépassant un simple stade fonctionnel, celui-ci établissant, par le choix des prestations retenues, une logique d'action de préservation de l'intérêt du client. Les dispositifs de jugement restent, somme toute, modestes, ce qui a pour conséquence de brider l'bomo singularis ${ }^{26}$ qu'est le consommateur effectuant le choix d'un EHPAD.

\section{Le risque d'une désingularisation des produits personnalisés}

La marchandisation de certains champs de l'action sociale et médico-sociale participe à l'économie des singularités. Mais à l'inverse, ne pourrait-elle aussi contribuer à une logique de désingularisation des produits personnalisés? «La désingularisation qualifie le processus, plus ou moins apparent, c'est-à-dire plus ou moins insaisissable, de conversion du produit symbolique qui transforme l'originalité en uniformité27. » Plusieurs facteurs peuvent jouer en ce sens : la mise en place de procédures visant à une standardisation des actes, les restrictions budgétaires et la non prise en compte du coût de l'humain, les politiques de management trop souvent dépendantes de gouvernance gestionnaire, la judiciarisation des rapports entre prestataires et client.

Les travailleurs sociaux ont toujours défini euxmêmes l'activité nécessaire à une bonne prise en charge de la personne et la façon de l'exercer, souvent fondées sur le sens de l'humain et sur une connaissance des publics, voire une approche intuitive de ces derniers. Mais, la recherche de l'efficience de l'action sociale et médico-sociale (rationalisation du travail social, division des tâches, individualisation de la relation d'accompagnement, désinstitutionalisation) en modifie l'approche philosophique et politique ancrée dans la culture historique de ce milieu, au point de penser que le sens de l'intervention sociale est aujourd'hui remis en cause dans ses fondements et ses valeurs. C'est une véritable crise d'identité de la professionnalité des travailleurs sociaux qui se manifeste avec pour corollaire les discours sur l'usure, la fatigue, l'impuissance, l'épuisement, la démotivation, le stress, le burnout, la non-reconnaissance de la qualité de l'engagement des personnels. Ces éléments se confondent avec le développement de contraintes protocolaires obligeant les personnels au respect d'actes codifiés ayant pour effet de standardiser la prise en charge. Pour la personne âgée, le lever et le coucher, la toilette et les soins seront minutés, le temps défini correspondant à un nombre d'actes standards obligatoires, censés répondre à ses besoins. L'objectif est de contraindre le travailleur social à davantage de prestations dans un temps donné. Sur le plan économique, cela se traduit par des compressions de personnels, des non-renouvellements de postes, des licenciements. L'établissement limite ainsi ses coûts de fonctionnement. Cette prégnance gestionnaire, exercée par les tutelles et relayée par les hiérarchies, dessert la dimension humaine des prestations personnalisées. Pas le temps de "s'attarder» à écouter, à parler, à passer un moment avec la personne, à être attentif à ses besoins et désirs. Cette logique n'est pas forcément rédhibitoire. Elle peut aussi révéler la capacité de certains professionnels à se sentir plus expérimentés et endurants face à ces pratiques. Elle peut les renforcer dans leur volonté de « résistance » à préserver une qualité humaine à l'accompagnement de la personne, à revendiquer cette qualité comme pivot de l'intervention sociale autour de laquelle vient se greffer une certaine technicité. Les travailleurs sociaux démontrent ainsi que l'action sociale et médicosociale ne peut entièrement se soumettre à des procédés de rationalisation (Etude CNRS, 2008) ${ }^{28}$, que la dimension humaine ne peut être oubliée au risque de voir se développer des formes de maltraitances institutionnelles et professionnelles.

L'adhésion à des normes, des procédures, des protocoles, des référentiels, comme l'inscription dans une gouvernance gestionnaire, marquée par un déficit de projet politique, sont aujourd'hui des marqueurs de mutation des pratiques du travail social, issus du monde économique. Ils se diffusent dans le champ social et médico-social : démarche qualité, évaluation, individualisation de la prise en charge, méthodologie de projet, contractualisation (Chauvière M., 2009) ${ }^{29}$. La pression managériale influence ainsi les professions dans le sens d'une rationalisation et d'une stan- 
dardisation des pratiques professionnelles. La judiciarisation du travail social participe à ces logiques, et doit être comprise comme une condition nécessaire à l'exercice du travail social et non comme une hégémonie du droit. Elle inscrit les pratiques professionnelles dans le respect des cadres légaux, c'est-à-dire dans un rapport de régulation sociale entre le professionnel et le client. L'application du droit relève d'une démarche réfléchie et mesurée. Ainsi, l'impératif légal, s'ajoutant à l'impératif gestionnaire, favorise la codification du rapport au client. Le cadre normatif peut être positif s'il ne donne pas lieu à un excès de contentieux, favorisant alors des pratiques de repli plutôt que d'innovation.

Tous ces facteurs peuvent jouer dans le sens d'une déshumanisation de la relation avec le client et favoriser une désingularisation du produit personnalisé. Ce sont les capacités d'adaptation des travailleurs sociaux à développer des pratiques qui sauront préserver les qualités humaines indispensables à une prise en charge cohérente et efficace de la personne. L'usagercitoyen, autre dimension du client, est également considéré au cœur de son dispositif de prise en charge.

\section{Conclusion}

Nombre d'interrogations restent sans réponse. En effet, comment déterminer le comportement rationnel, le comportement raisonnable du client? Comment déterminer au plus juste la meilleure réponse aux besoins de la personne prise en charge? Nombre d'indicateurs sont à inventer pour mesurer la satisfaction des besoins et désirs du client. En réponse à ces questions, l'économie des singularités fait référence au modèle de la rationalité procédurale. Ce modèle «a pour vertu de fixer les conditions de la comparaison afin de classer les degrés de rationalité de l'action et d'imposer le recours à l'analyse qualitative comme au calcul $^{30}$. $\gg$ Il suppose la rencontre entre le client et les dispositifs de jugement, puis la confrontation avec le produit personnalisé. Tel qu'il se pratique dans le cadre de l'action sociale et médico-sociale, ce modèle demeure encore trop aléatoire et incertain et peine à dissiper le caractère opaque du marché des singularités dans ce secteur. Cette opacité tient à une démocratisation $^{31}$ insuffisante de l'action sociale et médicosociale. L'application du droit des usagers le démontre, celui-ci restant très inégalement respecté, voire parfois ignoré. Ce droit n’a pas, à ce jour, constitué une révolution dans ce secteur, et encore moins à positionner le client dans son rôle de citoyen-acteur. L'application réelle des droits, comme expression d'une révolution culturelle des pratiques professionnelles, participera, sans nul doute, à l'assise d'une économie des singularités relevant le défi du choix cohérent et raisonnable dans le champ de l'action sociale et médico-sociale.

L'économie des singularités dans le secteur social et médico-social, et en particulier dans le champ de la prise en charge des personnes âgées, reste à ce jour immature. C'est une économie qui se construit, pour l'heure, de manière unilatérale. En effet, à ce jour, elle reste trop dépendante des seules autorités de tarification et des opérateurs. Aucun rapport de force n'établit quelle est la place du client dans ces enjeux économiques. La posture de client et les actions afférentes n'existent pas de manière collective. En cas d'insatisfaction, c'est individuellement que le client revendique. S'il existe des tentatives de regroupement associatif, celles-ci restent limitées et isolées. Mais, le rapport de force ne s'établit pas.

L'économie des singularités a le mérite d'ouvrir des champs de compréhension sur l'opérationnalité de la formulation du choix, inscrit dans un jeu complexe de l'estimation de la qualité. Elle permet de se questionner sur le type de régulation économique dans lequel les acteurs professionnels, comme les clients usagers de l'action sociale et médico-sociale souhaitent contracter et pérenniser leurs engagements. Mais, elle ouvre aussi un nouveau champ de questionnements éthiques. En effet, la logique des singularités pousse toujours plus loin l'individualisation de la personne. Dans le même temps, s'opère un transfert de charge de la responsabilité du groupe (l'institution, la société) vers l'individu. Une question sous-jacente émerge de fait. Qu'en sera-t-il des capacités de l'individu à se gérer? De nouveaux clivages sociaux vont apparaitre. Ils reposeront sur l'isolement de la personne, sur sa fragilité à accéder aux droits et les dispositifs d'accompagnement liés. Le risque sera grand de voir la personne adopter une posture de renoncement face à la difficulté de devoir se gérer. De nouveaux acteurs pour de nouveaux marchés ne manqueront pas de se positionner. L'aide à l'accompagnement a de beaux jours devant elle, mais à quel prix? 


\section{NOTES}

1 Les éléments constitutifs de la théorie néo-classique de l'économie sont toujours les mêmes quelle que soit la définition de celle-ci : l'acteur rationnel doté de préférences stables et exclusivement orienté par la recherche de la maximisation du profit ou de l'utilité; l'équilibre de l'offre et de la demande par la variation des prix et les états d'équilibre du marché fixés par les conditions de la concurrence (Karpik, 2007, p. 25). Bien que Karpik se garde de toute remise en cause de la théorie néolibérale, c'est bien par rapport à cette dernière que se construisent les éléments conceptuels de l'économie des singularités.

2 Ces établissements médicalisés accueillent des personnes âgées en perte d'autonomie physique ou psychique qui ne peuvent plus rester à leur domicile et qui ont besoin d'une assistance constante et d'une surveillance médicale.

3 L'auteur donne ici la définition de ce qu'il appelle des produits singuliers qui peuvent avoir un caractère tant individuel que collectif. Chaque produit peut amener une interprétation qui peut conduire à une requalification du produit.

4 L'opacité du marché repose sur l'incertitude de la qualité du produit, sur une information orientée en faveur du prestataire par exemple. La connaissance est donc l'élément central de l'objectivation du choix, alimentant lui-même la dynamique de marché (Karpik, 2007, p. 42).

5 Les dispositifs de jugement ont bien le rôle de dissiper l'opacité du marché (Karpik, 2007, p. 68). Le principe de fonctionnement des dispositifs de jugement vaut la délégation du consommateur à ces derniers. En effet, le consommateur s'en remet volontairement à un dispositif. C'est une part de sa liberté qu'il aliène à ce dispositif. C'est toute la dimension cognitive de l'établissement de la confiance qui lui est abandonnée (Karpik, 2007, p. 71).

6 L'Homo singularis appartient au marché des singularités comme l'Homo economicus appartient au marché standard. Il est soucieux de la qualité dont il cherche à établir les références.

7 Chaque dispositif de jugement est porteur d'une connaissance orientée. Chacun est choisi par le client selon une logique d'action identique. Le mécanisme de raréfaction nait d'une diversité plus grande des logiques d'action des consommateurs que l'univers des logiques d'action des dispositifs de jugement (Karpik, 2007, p. 102).

8 Le marché des biens et services singuliers révèle une multitude de goûts, d'opinions dont les dispositifs de jugement ne permettent pas d'établir une généralisation. C'est pourquoi il est utile d'établir un outil intermédiaire, le régime de coordination, qui permet de garantir une unité de point de vue et d'identifier les entités analytiques collectives qui permettent d'avoir une compréhension du marché des singularités (Karpik, 2007, p. 138).

9 La plupart des services et prestations assurés dans le secteur social et médico-social entrent dans ces régimes.

10 Ce type de réseau s'avère relativement faible dans le secteur social et médico-social.

11 Le marché-réseau constitue en somme une plateforme sur laquelle s’édifient les diverses architectures composant les marchés des services personnalisés (Karpik, 2007, p .235).

12 La RCB est une méthode datant des années soixante-dix qui fixe un cadre analytique au processus décisionnel des pouvoirs publics. Cette méthode a été abandonnée en 1984 comme procédure formalisée. La volonté de rationaliser les dépenses des ministères est reprise aujourd'hui par la loi organique relative aux lois de finances (LOLF). Cette rationalisation repose sur les concepts de mission, programme, performance, résultat.

13 «Client était le terme générique de l'action sociale d'avant les années 1975. Le terme fut remplacé par celui d’usager jugé plus valorisant dans un usage généralisé de services publics. Aujourd'hui, à travers la reprise de ce terme, on parle d'un service personnalisé en fonction des problèmes et des demandes de l'individu. Il traduit un renversement de la conception classique des institutions de travail social : le client devient roi, il n'est plus captif. Exigeant, il reste toutefois tributaire des différents services proposés, dans lesquels il a la possibilité de faire un choix de façon plus « éclairé ». Qui dit clientélisme dit aussi recherche d'un service de qualité afin de fidéliser ce client comme un autre » (Roux, 2002, p. 137).

14 Il est fort probable que, dans les temps à venir, sera élaboré un classement des établissements et services sociaux et médicosociaux comme existe déjà un classement des meilleurs hôpitaux de France.

15 Des cabinets privés libéraux d'assistantes sociales, d'éducateurs développent une offre de service personnalisée, à domicile ou en cabinet. L'argument principal de « vente » est la garantie d'un résultat. Reste à savoir, bien évidemment, ce que l'on peut entendre comme résultat, en matière d'intervention sociale.

16 La DGCS a pour mission de concevoir, piloter et évaluer les politiques publiques de solidarité, de développement social et de promotion de l'égalité, afin de favoriser la cohésion sociale et le soutien à l'autonomie des personnes.

17 La dotation globale de financement est égale à la différence entre la totalité des charges d'exploitation du budget auquel elle se rapporte, après incorporation le cas échéant du résultat d'un exercice antérieur et les produits d'exploitation du même budget autres que ceux relatifs à la dotation globale.

18 Le prix de journée est obtenu par la différence entre la totalité des charges d'exploitation du budget auquel il se rapporte, après incorporation le cas échéant du résultat d'un exercice antérieur et les produits d'exploitation du même budget autres que ceux relatifs au tarif journalier (ou prix de journée). Pour obtenir le prix de journée, cette différence est ensuite divisée par le nombre de journées. 
19 Circulaire du 18 janvier 2010 relative aux relations entre les pouvoirs publics et les associations : conventions d'objectifs et simplification des démarches relatives aux procédures d'agrément (JORF nº016du 20 janvier 2010, p. 1138, texte n¹).

20 Le produit personnalisé désigne le plus souvent un service qu’un bien. Il fait référence à la notion de service telle qu'elle est utilisée par des sociologues et des économistes. Pour la sociologie, la « relation de service » se confond avec le service personnalisé; elle porte sur une activité marquée par l'incertitude sur la qualité et par l'incertitude sur les résultats (Karpik, 2007, p. 50).

21 Une prise en charge personnalisée assurée par un ensemble de «professions » et de métiers : aide médico-pédagogique, assistante sociale, infirmière, médecin, psychologue ...

22 «Qu’il soit local ou général, inhabituel ou routinier, le jugement exprime un point de vue particulier. Il construit la relation adéquate entre des critères d'évaluation dont la composition et la pondération relative sont variables et le cadrage de la situation, c'est-à-dire l'organisation des faits. Le jugement associe la valeur et la connaissance. Il intègre dans une décision synthétique nécessairement arbitraire, l'hétérogénéité des critères et l'hétérogénéité des mondes. Il fonde la comparaison des incommensurables » (Karpik, 2007, p. 65).

23 «La morale professionnelle et la fierté professionnelle manifestent l'existence de deux catégories de dispositifs qui fonctionnent différemment : l'une impose les mêmes devoirs à tous, alors que l'autre justifie l'hétérogénéité relative des pratiques. L'une est un mode de protection de la similitude, l'autre une garantie de la diversité. » (Karpik, 2007, p. 256).

24 L'Agence Nationale de l'Évaluation et de la qualité des établissements et services sociaux et médico-sociaux (ANESM) a été intégrée à la Haute Autorité de Santé en date du 1er avril 2018 (article 72 de la LFSS). En mai 2011, l'ANESM a publié des recommandations de bonnes pratiques intitulées : «Qualité de vie en EHPAD (Volet 1) : De l'accueil de la personne à son accompagnement. L'objectif de cette recommandation est de donner des pistes de réflexion et d'action aux professionnels pour améliorer la qualité de vie des personnes entre le moment de la demande d'admission et les premiers mois dans l'établissement. Plus précisément, il s'agit de renforcer l'importance de la préparation à l'entrée et de la période d'accueil pour accueillir au mieux la personne dans son nouveau lieu de vie, mettre en place la personnalisation de l'accompagnement et des prestations, au moyen notamment du projet personnalisé, de favoriser l'ouverture de l'EHPAD vers l'extérieur et d'en améliorer la perception par le grand public, la population du territoire, les familles et les personnes âgées qui seront susceptibles d'y finir leurs jours. Ces recommandations seront complétées par d'autres volets : volet 2, le cadre de vie et la vie quotidienne; volet 3, la vie sociale des résidents; volet 4, l'impact des éléments de santé sur la qualité de vie.

25 «Les dispositifs de jugement ne sont pas des machines à produire de la transparence entre des produits et des consommateurs dont les qualités et les préférences préexisteraient à leurs interventions. Ils proposent une connaissance orientée et, implicitement et explicitement, ils fixent les conditions que le consommateur doit respecter pour que l'ajustement du produit et du consommateur soit satisfait. Ils qualifient simultanément le produit et le client » (Karpik, 2007, p. 77).

26 Certes, l'homo singularis du secteur social et médico-social n'a pas les pleines qualités d'un agent rationnel qui s'autodétermine après avoir déterminé les fins qu’il vise et avoir délibéré sur les moyens nécessaires à les atteindre (Marzano, 2018, p. 95). Mais, il peut y être aidé par les ayant-droit et les professionnels de sa prise en charge dans le respect de sa qualité de citoyen.

27 La désingularisation cristallise la perte d'une valeur affirmée du bien singulier. Cette perte peut être liée à l'expression de réticences, de doutes, de prises de position, de critiques qui peuvent se traduire en réclamations, en dénonciations, en mobilisations, en recours (Karpik, 2007, p. 317).

28 Étude intitulée «Usure des travailleurs sociaux et épreuve de professionnalité. Les configurations d'usure : clinique de la plainte et cadres d'action contradictoires », menée de février 2007 à mai 2008 par des chercheurs (Bertrand Ravon, Geneviève Decrop, Jacques Ion, Christian Laval, Pierre Vidal-Naquet), laboratoire Mondes et dynamiques des sociétés (CNRS) en collaboration avec l’observatoire national des pratiques en santé mentale et précarité, commanditaire Observatoire nationale de la pauvreté et de l'exclusion sociale, permet de distinguer schématiquement trois types de parcours d'épreuves. D'une part, l'analyse de certains parcours montre des professionnels dotés d'un solide self-control et d'un statut professionnel bien établi, sujets de leurs pratiques. Adossés à leurs institutions d'exercice, mais remontés contre elles quand celles-ci abandonnent les valeurs fondatrices, c'est dans l'adversité à leurs tutelles qu'ils arrivent à trouver l'énergie nécessaire au maintien de leur action. D'autre part, on peut observer des intervenants moins stabilisés dans un statut professionnel fort, plus engagés dans les situations, travaillant davantage leur subjectivité au risque de perdre quelque peu la maitrise de la situation, mais qui, par les nombreuses voies qu'ouvre paradoxalement l'indétermination des rencontres, peuvent forger une professionnalité innovante, quand bien même fragile. Enfin, mais il est par définition beaucoup plus difficile de les rencontrer, on peut étudier des parcours de professionnels épuisés dont la plainte ne remonte pas du terrain, du fait même de leur isolement et de leur absence de reconnaissance, du fait même aussi de l'absence d'une reprise collective et adaptée de leurs difficultés.

29 Voir à ce sujet, Chauvière, M. (2009). Le travail social à l'épreuve du management et des impératifs gestionnaires. Colloque organisé par l’Université Toulouse II, Le Mirail, 1 au 3 juillet 2009.

30 L'effectivité de la rationalité procédurale repose sur la rencontre de l'acteur et des dispositifs de jugement et celle du consommateur et le produit désiré (Karpik, 2007, p. 109).

31 La démocratisation peut reposer sur une augmentation des dispositifs de jugements. L'accès pour les usagers ou clients aux résultats des évaluations internes et externes renforcerait ces dispositifs. 


\section{RÉFÉRENCES}

Auzoux, D. (1997). Le management de la qualité et les normes ISO. Les cahiers de l'Actif, 256/257, 89-96.

Repéré à http://perso.wanadoo.fr/actif-online/portail/auzoux.html

Batifoulier, F. (2011). Manuel de direction en action sociale et médico-sociale. Paris, France : Dunod.

Chauvière, M. (2009). Qu'est-ce que la « chalandisation »? Informations sociales. 2(152), 128-134. Repéré à https://www.cairn.info/revue-informations-sociales-2009-2-page-128.htm.

Defontaine, J. (2005). La marchandisation des actions sociales et médico-sociales. Paris, France : édition ASH, collection ASH professionnels.

Defontaine, J., Jaeger, M., Ledoux, H. et Sarazin, I. (2005). Loi 2002-2 (2005) : logique de marché et/ ou de promotion de la citoyenneté? Paris, France : Édition ASH, 25-28.

Dujarier, A.M. (2014). Le travail du consommateur, de Mac Do à eBay : comment nous coproduisons ce que nous achetons. Paris, France : La Découverte/Poche, Essais n 404.

Étude CNRS, (2008). Usure des travailleurs sociaux et épreuve de professionnalité. Les configurations d'usure : clinique de la plainte et cadres d'action contradictoires, menée de février 2007 à mai 2008, par des chercheurs (Bertrand Ravon, Geneviève Decrop, Jacques Ion, Christian Laval, Pierre Vidal-Naquet), laboratoire Mondes et dynamiques des sociétés (CNRS) en collaboration avec l'observatoire national des pratiques en santé mentale et précarité, commanditaire Observatoire nationale de la pauvreté et de l'exclusion sociale.

Gacoin, D. (2007). L'arrivée des bonnes pratiques professionnelles : dynamisation ou standardisation. Idées, concepts \& auteurs. Repéré à https://danielgacoin.blogs.com/blog/2007/02/larrive_des_bon.html

Gadrey, J. (1994). Les relations de service dans le secteur marchand. Dans J. Gadrey et Bandt, J. Relations de services, marché de services (pp. 23-41) Paris, France : CNRS.

Gautié, J. (2008). Peut-on faire l'économie des singularités? Revue française de sociologie. 49(2), 391-406. Repéré à https://www.cairn.info/revue-francaise-de-sociologie-1-2008-2-page-391.htm

Karpik, L. (2007). L'économie des singularités. Bibliothèque des sciences humaines. Paris, France : NRF- Éditions Gallimard.

Laurent, S. (2010). Subventions aux associations et réglementation européenne : les clarifications de la circulaire Fillon. Site Association1901.fr. Repéré à http://bit.ly/cxTfuK.

Marzano, M. (2018). L'éthique appliquée. Paris, France : Que sais-je?

Roux, M.A. (2002). Promouvoir la place de l'usager : d'une vision mécaniste des professions du social vers un accompagnement moderniste et politique du citoyen. Les cabiers de l'Actif, 318/319, 137-148.

Zalio, P.-P. (2007, 23 novembre). L'économie des biens singuliers. La Vie des idées. Repéré à http://www.laviedesidees.fr/ L-economie-des-biens-singuliers.html 